\title{
Laminins in an in vitro anterior lens capsule model established using HLE B-3 cells
}

\author{
YU YAN ${ }^{1,2}$, HUA QIAN ${ }^{2}$, HONGDA JIANG $^{3}$, HAIYANG YU $^{1,2}$, LIYAO SUN $^{1,2}$, \\ XI WEI $^{1,2}$, YUNDUAN SUN ${ }^{1}$, HONGYAN GE ${ }^{1}$, HAIZHOU ZHOU ${ }^{3}$, XIAOGUANG LI $^{2}$, \\ TAKASHI HASHIMOTO ${ }^{4}$, XIANLING TANG ${ }^{1,5}$ and PING LIU ${ }^{1,5}$
}

${ }^{1}$ Eye Hospital, First Affiliated Hospital, Harbin Medical University, Harbin, Heilongjiang 150001;

${ }^{2}$ Academician Workstation, Harbin Medical University and Heilongjiang Academy of Medical Sciences, Harbin,

Heilongjiang 150081; ${ }^{3}$ Department of Laboratory Diagnosis, First Affiliated Hospital, Harbin Medical University, Harbin, Heilongjiang 150001, P.R. China; ${ }^{4}$ Department of Dermatology, Osaka City University Graduate School of Medicine, Osaka 545-8585, Japan; ${ }^{5}$ Eye Bank of Heilongjiang Province, Harbin, Heilongjiang 150001, P.R. China

Received August 8, 2017; Accepted January 11, 2018

DOI: $10.3892 / \mathrm{mmr} .2018 .8581$

\begin{abstract}
Cataracts are the most common eye disease to cause blindness in patients. The abnormal deposition of laminins (LMs) in the lens capsule and the disruption of capsular epithelium contribute to cataract development, although the mechanism by which this occurs is currently unclear. The present study aimed to reproduce HLE B-3 basement membranes (BMs) using HLE B-3 cells and to analyze the similarities of LM expression between HLE B-3 BMs and human anterior lens capsule (ALC). Immunohistochemistry (IHC), ELISA, western blot analysis and immunoprecipitation (IP)-western blot analysis were used to detect total LMs, LM trimers and 11 LM subunits in HLE B-3 cells, HLE B-3 BMs and human ALCs. In IHC staining, HLE B-3 cells and human ALCs were positive for LMs. In LM ELISA, all samples analyzed were positive for LMs. Western blot analysis detected all LM subunits except for LM $\gamma 3$ in HLE B-3 cell lysate, 4 subunits (LM $\alpha 4, \mathrm{LM} \alpha 2, \mathrm{LM} \alpha 1$ and $\mathrm{LM} \gamma 1)$ in HLE B-3 cell culture supernatant, 5 subunits ( $\mathrm{LM} \alpha 4, \mathrm{LM} \alpha 2, \mathrm{LM} \alpha 1$, LM $\beta 3$ and LM $\gamma 1$ ) in HLE B-3 BMs, and 3 subunits (LM $\alpha 4$, $\mathrm{LM} \gamma 2$ and $\mathrm{LM} \gamma 1$ ) in human ALCs. The results of IP-western
\end{abstract}

Correspondence to: Dr Ping Liu or Dr Xianling Tang, Eye Hospital, First Affiliated Hospital, Harbin Medical University, 23 Youzheng Street, Harbin, Heilongjiang 150001, P.R. China

E-mail: liuping20171q@163.com

E-mail: tangxianling2007@126.com

Abbreviations: ALC, anterior lens capsule; BM, basement membrane; H\&E staining, hematoxylin and eosin staining; ICC, immunocytochemistry; IHC, immunohistochemical staining; IP, immunoprecipitation; LECs, lens epithelial cells; LM, laminin; $\mathrm{mAb}$, monoclonal antibodies; $\mathrm{pAb}$, polyclonal antibodies

Key words: anterior lens capsule, basement membrane, cataract, human lens epithelial cells, laminin, trimer blot analysis revealed that the LM411 trimer was detected in HLE B-3 cell culture supernatant. These results indicated that HLE B-3 BMs were similar to human ALCs in terms of LM expression. Therefore, HLE B-3 BMs could be used as an in vitro ALC model to determine the role of LMs in ALC in the pathogenesis of cataracts and to select potential anti-cataract drugs.

\section{Introduction}

A cataract is defined as any type of opacity of the eye lens that affects vision (1). Cataracts are the main cause of blindness and visual impairment worldwide, particularly in developing countries $(2,3)$. There are a number of risk factors for the development of cataracts, including ultraviolet light exposure, diabetes, aging and other environmental factors (4-6). Despite the number of studies that have been conducted on cataracts, their pathogenesis remains unknown. Thus, effective non-surgical therapies are currently lacking, which is a substantial burden to healthcare budget (7). Dysfunction of lens epithelial cells (LECs) has been repeatedly proposed to serve a key role in cataract formation, although this mechanism is unknown (8-10).

Laminins (LMs) are large-molecular-weight trimer glycoproteins consisting of $\alpha-, \beta$ - and $\gamma$-subunits, which integrate in almost all basement membranes (BMs), functioning as scaffolds (11). It has been suggested that LM trimers are assembled inside the cell, with extracellular proteolytic processing of various subunits leading to the mature LMs (12). In addition to their function as scaffold of BMs, LMs can also interact with cell surface receptors such as intergrins to control signaling events that regulate cellular proliferation, migration and differentiation $(13,14)$.

Particular LM isoforms are present in specific tissue and cell distributions during developmental and pathological conditions, indicating that different LM isoforms have distinct functional roles $(12,14)$. Past studies have demonstrated that $\mathrm{LM} \alpha 1, \mathrm{LM} \alpha 5, \mathrm{LM} \beta 1, \mathrm{LM} \beta 2$ and $\mathrm{LM} \gamma 1$ subunits exist in the 
developmental human lens capsule $(15,16)$. Another prior study demonstrated that human adult lens capsule is composed of $\mathrm{LM} \alpha 1, \mathrm{LM} \alpha 2, \mathrm{LM} \alpha 3, \mathrm{LM} \alpha 4, \mathrm{LM} \alpha 5, \mathrm{LM} \beta 1, \mathrm{LM} \beta 2$ and $\mathrm{LM} \gamma 1$ subunits, and the predominant LM trimer was LM521 (17).

Gene defects and the abnormal expression of LMs are closely associated with various lens diseases (18-23). LM 32 mutations cause Pierson syndrome, which has various ocular lesions, including abnormal lens shape, posterior lenticonus and cataracts (19). It has been demonstrated that development of LM-positive linear capsular densities and occurrence lens epithelial degenerative alterations are associated with aging (20). Increased LM expression underneath lens capsules and abnormal LEC migration were identified in FE65/FE65L1 double knockout lenses, which may be involved in the formation of cortical cataracts (21). Abnormal accumulation of LMs in the lens capsule and epithelial-mesenchymal transition of LECs was also observed in patients with anterior subcapsular opacification and posterior capsule opacification $(22,23)$.

Human primary LECs in long-term cultures could synthesize a continuous sheet of capsule-like material (24). Theoretically, human LEC lines could also produce anterior-capsule-like BM with rich LMs in vitro, which could be a suitable disease model to study the role of LMs in anterior lens capsule (ALC) in the pathogenesis of lens diseases, particularly cataracts.

In the present study, the human LEC HLE B-3 line was used to construct the BMs and further analyze the expression levels of 11 LM subunits. Similarities in LM expression between HLE B-3 BMs and human ALCs were also evaluated. The results of the present study may aid in elucidating the role of LMs in ALC and cataract pathogenesis.

\section{Materials and methods}

HLE B-3 cell culture and preparation of cell lysate and culture supernatant. The human LEC HLE B-3 line were obtained from the American Type Culture Collection (Manassas, VA, USA) and cultured in low-glucose DMEM (Hyclone; GE Healthcare Life Sciences, Logan, UT, USA) containing 10\% fetal bovine serum (FBS) (Gibco; Thermo Fisher Scientific, Inc., Waltham, MA, USA), $100 \mathrm{U} / \mathrm{ml}$ penicillin and $100 \mu \mathrm{g} / \mathrm{ml}$ streptomycin (Beyotime Institute of Biotechnology, Haimen, China) at $37^{\circ} \mathrm{C}$ in humidified atmosphere with $5 \% \mathrm{CO}_{2}$. Cells were seeded in $10-\mathrm{cm}$ dishes and grown to $95 \%$ confluence. Following discarding the culture supernatant, cells were washed twice with PBS and lysed with cold radioimmunoprecipitation assay (RIPA) buffer [50 mM Tris- $\mathrm{HCl}$ (pH 7.5), $150 \mathrm{mM} \mathrm{NaCl}$, $1 \%$ Triton $\mathrm{X}-100$ and $1 \%$ protease inhibitor cocktail from Sigma-Aldrich; Merck KGaA, Darmstadt, Germany] at $4^{\circ} \mathrm{C}$ for $30 \mathrm{~min}$. Following centrifugation at $14,000 \times \mathrm{g}$ at $4^{\circ} \mathrm{C}$ for $20 \mathrm{~min}$, the supernatant was harvested and kept as cell lysate. When $95 \%$ confluent, cells were washed twice with PBS and cultured in $5 \mathrm{ml}$ low-glucose DMEM without FBS for $48 \mathrm{~h}$. The cell supernatant was harvested and centrifuged at $3,000 \mathrm{xg}$ at $4^{\circ} \mathrm{C}$ for $5 \mathrm{~min}$ to remove debris.

Immunocytochemical staining (ICC) of HLE B-3 cells. For hematoxylin and eosin (H\&E) staining, HLE B-3 cells grown on glass slides were stained with hematoxylin and eosin (5 and 2 min, respectively, at room temperature), and examined under a light microscope (Olympus Corporation, Tokyo, Japan; magnification, x400). For ICC of LMs, HLE B-3 cells grown on glass slides were incubated for $10 \mathrm{~min}$ at room temperature with $3 \%$ hydrogen peroxide, and blocked with $10 \%$ normal goat serum for $20 \mathrm{~min}$ at room temperature, followed by incubation for $60 \mathrm{~min}$ at room temperature with rabbit anti-LM antibody (1:200; cat. no. ab11575; Abcam, Cambridge, UK). Subsequently, samples were incubated for $30 \mathrm{~min}$ at room temperture with peroxidase conjugated anti-rabbit antibody (cat. no. PV-9000; OriGene Technologies, Inc., Beijing, China) without dilution and color development was performed with 3,3'-diaminobenzidine (DAB). Staining was visualized with a light microscope (Nikon TE300, Nikon Corporation, Tokyo, Japan; magnification, $\mathrm{x} 400$ ), and images were captured with an attached digital camera and associated software (SPOT Basic ${ }^{\mathrm{TM}}$ image capture software; cat. no. SPOT53BE; SPOT Imaging, a division of Diagnostic Instruments, Inc., Sterling Heights, MI, USA).

BM preparation using HLE B-3 cells. When HLE B-3 cells reached $95 \%$ confluence, they were washed with PBS and cultured in fresh low-glucose DMEM medium with 5\% FBS; this day was recorded as day 0 . At days $0,3,6,9$ and 12, HLE B-3 BMs were harvested, as described previously $(25,26)$. Briefly, HLE B-3 cells were washed with PBS four times, after which $0.03 \%$ ammonia and $0.1 \%$ Triton-PBS was added to remove HLE B-3 cells completely. Finally, HLE B-3 BM remained on the bottom of the dish, which was washed with double distilled water 4 times and harvested with $150 \mu 1$ of $4 \mathrm{X}$ sample buffer [ $8 \%$ sodium dodecyl sulfate, $0.2 \mathrm{M}$ Tris- $\mathrm{Cl}$ (pH 6.8), 40\% glycerol, $0.2 \%$ bromophenol blue and $0.1 \mathrm{M}$ dithiothreitol] at room temperature or $1 \mathrm{ml}$ RIPA buffer on ice.

Human ALCs and protein lysates. Human ALCs were obtained from autopsies from 3 individuals (1 female and 2 male) aged 25-79 years old from November to December of 2016 at the Eye Bank of Heilongjiang Province (Harbin, China). Human ALCs were obtained by a single ophthalmologist using central circular capsulorhexis method from anterior surface of transparent lens (27). All experiments were performed with the approval of the Internal Review Board of Harbin Medical University and were conducted in accordance with Declaration of Helsinki Principles. Informed consent was obtained from all patients prior to mortality or from their families following mortality for inclusion of autopsy data. Samples were harvested within 4-24 h of mortality. Human ALCs were dissected from the isolated transparent lenses and were lysed with cold RIPA buffer at $4^{\circ} \mathrm{C}$ overnight. Finally, the supernatant was kept as human ALC lysate following centrifugation at $14,000 \mathrm{xg}$ at $4^{\circ} \mathrm{C}$ for $20 \mathrm{~min}$.

$H \& E$ staining and immunohistochemistry (IHC) staining of human ALCs. Following careful dissection from transparent lenses, excised human ALCs were frozen at $-80^{\circ} \mathrm{C}$. Frozen human ALC tissues were transversely sectioned at $5-\mu \mathrm{m}$ thickness, mounted on glass slides, fixed with cold acetone for $15 \mathrm{~min}$ at $4^{\circ} \mathrm{C}$, followed by $\mathrm{H} \& \mathrm{E}$ staining or IHC with identical steps to ICC, as aforementioned. 
Bicinchoninic acid assay. Protein concentrations in the HLE B-3 cell lysate, HLE B-3 cell culture supernatant, human ALC lysate and various HLE B-3 BMs were measured using a Bicinchoninic Acid Assay kit (Beyotime Institute of Biotechnology) according to the manufacturer's protocol.

ELISA. The total LM levels were measured by using commercially available LM ELISA kit in accordance with the manufacturer's protocol (E-EL-H0128c; Elabscience, Houston, TX, USA). The antibodies used in this kit were polyclonal antibodies (pAbs) against all types of $\mathrm{LM} \alpha, \mathrm{LM} \beta$ and $\mathrm{LM} \gamma$ subunits.

Antibodies. Goat pAbs against LM $\alpha 5$ and LM $\gamma 3$ (1:200, cat nos. sc-16592 and sc-133178; Santa Cruz Biotechnology, Inc., Dallas, TX, USA), rabbit pAbs against LM $\alpha 4$, LM $\beta 3$ and LM 22 (1:1,000, cat nos. C13067, C13071 and C30224; Assay Biotechnology Company, San Francisco, California), rabbit pAbs against LM $\gamma 1$ (1:1,000, cat nos. ab69632; Abcam, Cambridge, UK), mouse monoclonal antibodies (mAb) against $\mathrm{LM} \alpha 4, \mathrm{LM} \alpha 3, \mathrm{LM} \alpha 2, \mathrm{LM} \alpha 1, \mathrm{LM} \beta 2$ and $\mathrm{LM} \beta 1$ (1:200, cat nos. sc-130540, SC-13586, sc-55605, sc-74418, sc-133241 and sc-17763; Santa Cruz Biotechnology, Inc.) were used in the present study.

Western blotting analysis. Five known LM $\alpha$ chains, three known LM $\beta$ chains and three known LM $\gamma$ chains in HLE B-3 cell lysate, HLE B-3 cell culture supernatant and HLE B-3 BMs harvested at days 0, 3, 6, 9 and 12, and human ALC lysate were analyzed by western blotting, as described previously (28). Briefly, antigen sources inclding HLE B-3 cell lysate, culture supernatant or protein lysate of human ALCs were mixed with $2 \mathrm{X}$ sample buffer, boiled for $2 \mathrm{~min}$ and separated by SDS-PAGE. The separated proteins were transferred to a polyvinylidene difluoride membrane (EMD Millipore, Billerica, MA, USA). Following blocking with $5 \%$ skimmed milk in Tris-buffered saline containing $0.05 \%$ Tween-20 (TBS-T) for $1 \mathrm{~h}$ at room temperature, membranes were incubated with the aforementioned primary antibodies diluted in solution 1 (Toyobo Life Science, Osaka, Japan) at $4^{\circ} \mathrm{C}$ overnight. Following washes with TBS-T, membranes were incubated with HRP-conjugated goat anti-mouse IgG (1:5,000; cat no. 31437; Thermo Fisher Scientific, Inc.), HRP-conjugated rabbit anti-goat IgG (1:5,000; cat no. 31433; Thermo Fisher Scientific, Inc.) or HRP-conjugated goat anti-rabbit IgG (1:5,000; cat no. 31463; Thermo Fisher Scientific, Inc.) diluted in solution 2 (Toyobo Life Science) for $1 \mathrm{~h}$ at room temperature. Under identical experimental conditions, normal rabbit IgG, normal mouse IgG or normal goat IgG (all 1:200; cat nos. sc-2027, sc-2025 and sc-2028; Santa Cruz Biotechnology, Inc.) were used as isotype controls. Finally, the antibody-antigen complex was visualized using an Enhanced Chemiluminescent kit (Beyotime Institute of Biotechnology).

Immunoprecipitation (IP)-western blot analysis. All subsequent procedures were performed at $4^{\circ} \mathrm{C}$, unless otherwise stated. A total of $1 \mathrm{ml}$ HLE B-3 cell culture supernatant was incubated overnight on a shaker with protein $G$ agarose (Beyotime Institute of Biotechnology) and anti-LM $\alpha 4$ rabbit
$\mathrm{pAb}$ or anti-LM $\gamma 1$ rabbit $\mathrm{pAb}$. The same concentrations of normal rabbit $\operatorname{IgG}$ were used as an isotype control for IP. Precipitates, collected by centrifugation at $3,000 \mathrm{x}$ g at $4^{\circ} \mathrm{C}$ for $2 \mathrm{~min}$, were washed three times with PBS containing $0.5 \%$ Triton $\mathrm{X}-100$, and were eluted from protein $\mathrm{G}$ agarose by boiling with $4 \mathrm{X}$ sample buffer for $2 \mathrm{~min}$. Proteins in the supernatant were separated by SDS-PAGE and detected by western blotting, as aforementioned.

\section{Results}

LMs in HLE B-3 cells. To investigate whether HLE B-3 cells were positive for LMs, H\&E staining and ICC for LMs were performed. As depicted in Fig. 1A and B, LMs were widely expressed in the cytoplasm of HLE B-3 cells. To investigate the expression of LMs in HLE B-3 cell lysate and culture supernatant, ELISA and western blot analysis was performed. ELISA for the presence of LMs generated positive results in HLE B-3 cell lysate and culture supernatant (Fig. 1C). By western blotting, all $11 \mathrm{LM}$ subunits were probed in the HLE B-3 cell lysate and culture supernatant. The HLE B-3 cell lysate was positive for $\mathrm{LM} \alpha 5, \mathrm{LM} \alpha 4, \mathrm{LM} \alpha 3, \mathrm{LM} \alpha 2, \mathrm{LM} \alpha 1, \mathrm{LM} \beta 3$, $\mathrm{LM} \beta 2, \mathrm{LM} \beta 1, \mathrm{LM} \gamma 2$ and $\mathrm{LM} \gamma 1$ subunits, whereas HLE B-3 cell culture supernatant was positive for $\mathrm{LM} \alpha 4, \mathrm{LM} \alpha 2, \mathrm{LM} \alpha 1$ and LM $\gamma 1$ subunits (Fig. 1D). The 10-fold concentrated HLE B-3 cell culture supernatant was also used for detection of 11 LM subunits by western blotting, and the results were identical to those obtained using the non-concentrated supernatant (data not shown).

LM trimers in HLE B-3 cell culture supernatant. To investigate what type of LM trimers appeared in HLE B-3 cell culture supernatant, IP experiments with antibodies against LM $\alpha 1-\mathrm{LM} \alpha 5$ for IP, and antibodies against LM $\beta 1-\mathrm{LM} \beta 3$ and $\mathrm{LM} \gamma 1-\mathrm{LM} \gamma 3$ for western blotting were performed. The immunoprecipitate obtained with antibodies for $\mathrm{LM} \alpha 1, \mathrm{LM} \alpha 2$, $\mathrm{LM} \alpha 3$ and LM $\alpha 5$ were negative in western blotting with antibodies for all LM $\beta$ and LM $\gamma$ subunits (data not shown). The immunoprecipitate obtained with antibodies for LM $\alpha 4$ was positive in western blotting with antibodies for LM $\beta 1$ and LM $\gamma 1$ (Fig. 2A), indicating the presence of the LM411 trimer in the HLE B-3 cell culture supernatant. To confirm this result, the immunoprecipitate obtained with antibodies for LM $\gamma 1$ was also analyzed by western blotting using antibodies against $\mathrm{LM} \alpha 4$ and LM $\beta 1$, which proved that LM $\gamma 1$ immunoprecipitates contained LM $\alpha 4$ and LM $\beta 1$ (Fig. 2B). These results confirmed that the LM411 trimer existed in the HLE B-3 cell culture supernatant.

LMs in HLE B-3 BMs. HLE B-3 BMs were harvested at days 0, $3,6,9$ and 12, and were analyzed for the expression of LMs by ELISA and western blotting. In LM ELISA, total LM levels in HLE B-3 BMs were similar at days 0, 3 and 6, but decreased to $70 \%$ at days 9 and 12 (Fig. 3A). Western blotting of HLE B-3 BMs detected the presence of $\mathrm{LM} \alpha 4, \mathrm{LM} \alpha 2, \mathrm{LM} \alpha 1, \mathrm{LM} \beta 3$ and LM $\gamma 1$ subunits (Fig. 3B). Expression levels of LM $\alpha 4$, LM $\alpha 2$ and LM $\alpha 1$ in HLE B-3 BMs were relatively higher at days 3 and 6 . LM $\beta 3$ expression levels increased with culture time. LM $\gamma 1$ expression levels fluctuated with time irregularly, with the lowest levels at day 12 . 
H\&E staining of HLE B-3 cells

A

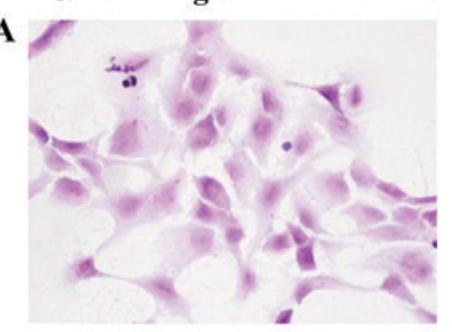

ICC of LMs in HLE B-3 cells

B

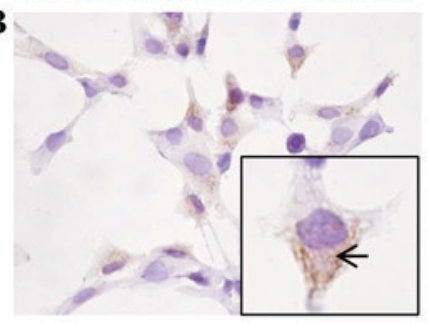

LM ELISA of HLE B-3 cells

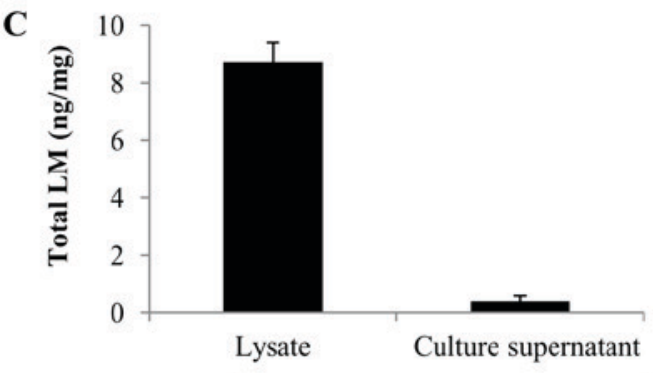

HLE B-3 cells

Western blotting of 11 LM subunits in HLE B-3 cells

D
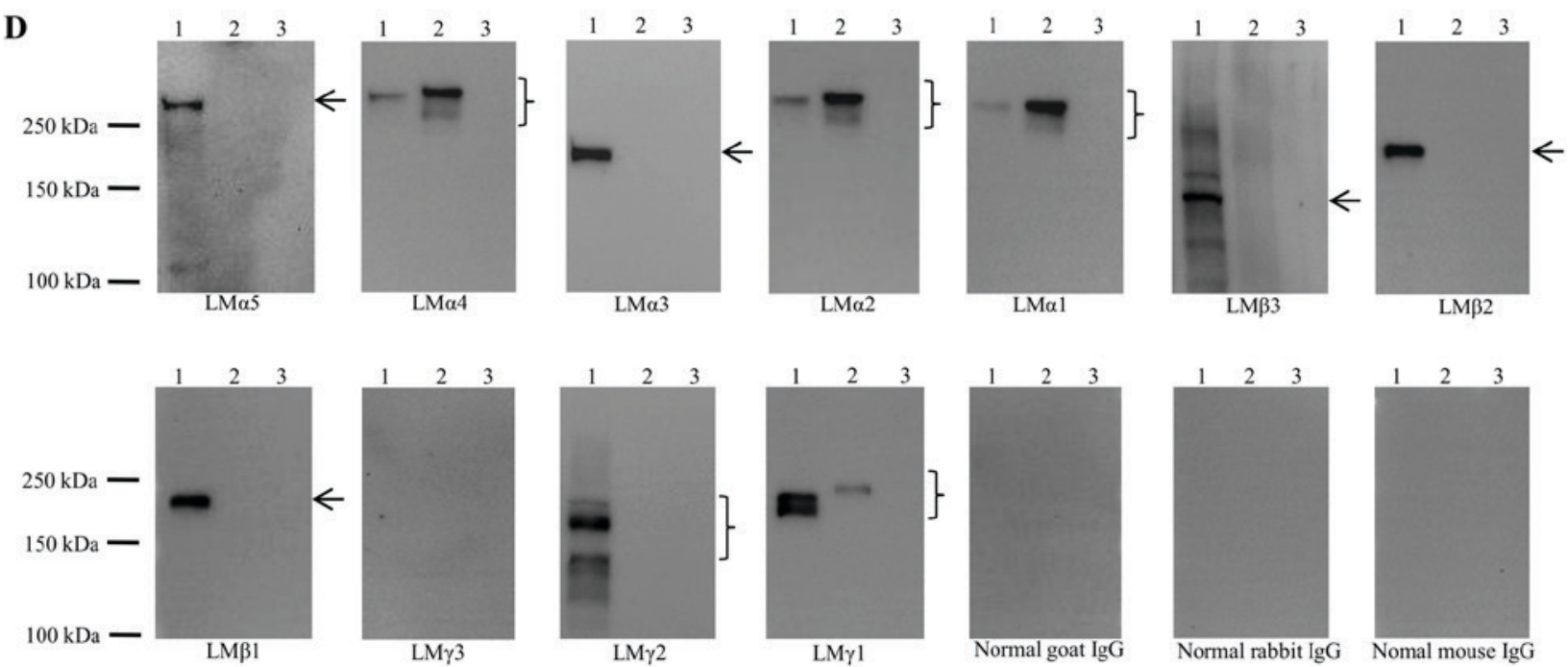

Figure 1. LMs in HLE B-3 cells. (A) H\&E staining of HLE B-3 cells (magnification, $x 400$ ). (B) ICC of LMs in HLE B-3 cells (magnification, $x 400$ ). (C) LM ELISA in HLE B-3 cells. (D) Detection of 11 LM subunits in HLE B-3 cells by western blotting. 1, HLE B-3 cell lysate; 2, culture supernatant; 3, culture medium. Arrows and braces indicate the target proteins. H\&E, hematoxylin and eosin; ICC, immunocytochemistry; LM, laminin.

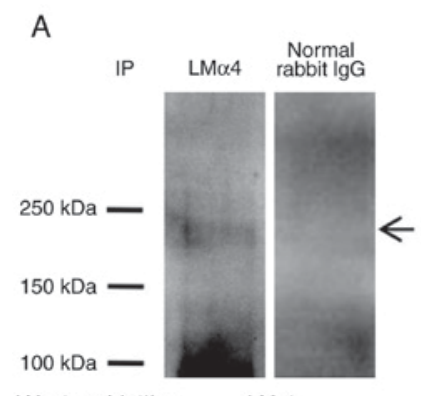

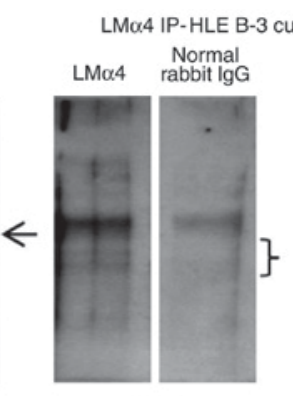

LMß1

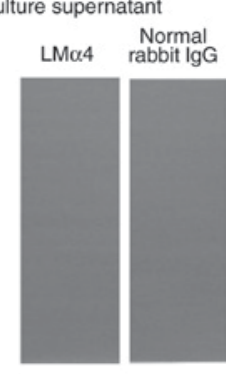

LMß2

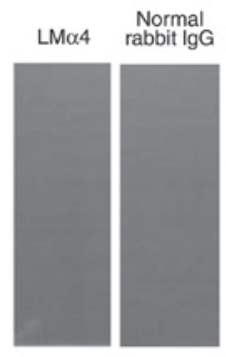

LMß3

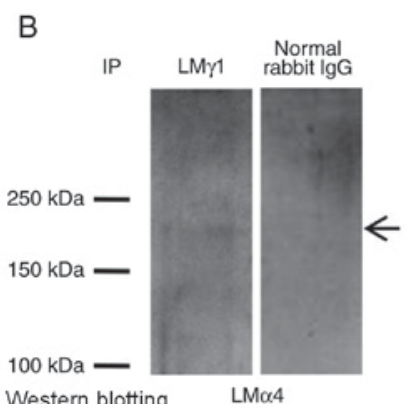

LMY1 IP-HLE B-3 culture supernatant
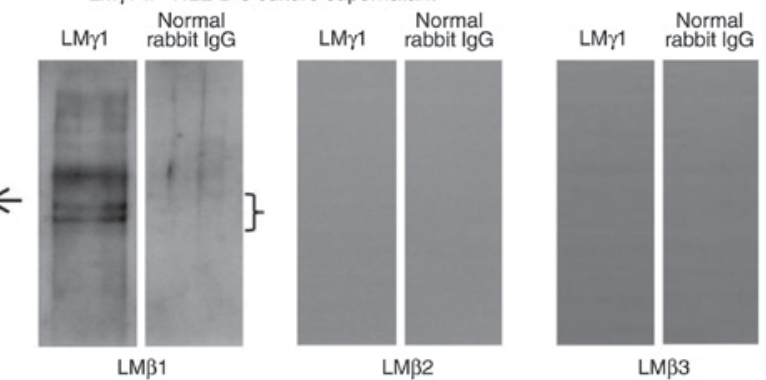

Figure 2. LM trimers in HLE B-3 cell culture supernatant. (A) Detection of LM trimers in HLE B-3 cell culture supernatant by LM $\alpha 4$ IP. An antibody against $\mathrm{LM} \alpha 4$ was used to precipitate LM trimers and the products were analyzed by western blotting using antibodies against LM $\beta 1$-LM $\beta 3$ and LM $\gamma 1$. (B) Detection of LM trimers in HLE B-3 cell culture supernatant by LM $\gamma 1$ IP. An antibody against LM $\gamma 1$ was used to precipitate LM trimers and the products were analyzed by western blotting using antibodies against LM $\beta 1$-LM 33 and LM $\alpha 4$. Arrows and braces indicate the target proteins. IP, immunoprecipitation; LM, laminin. 


\section{LM ELISA of HLE B-3 BM}

A

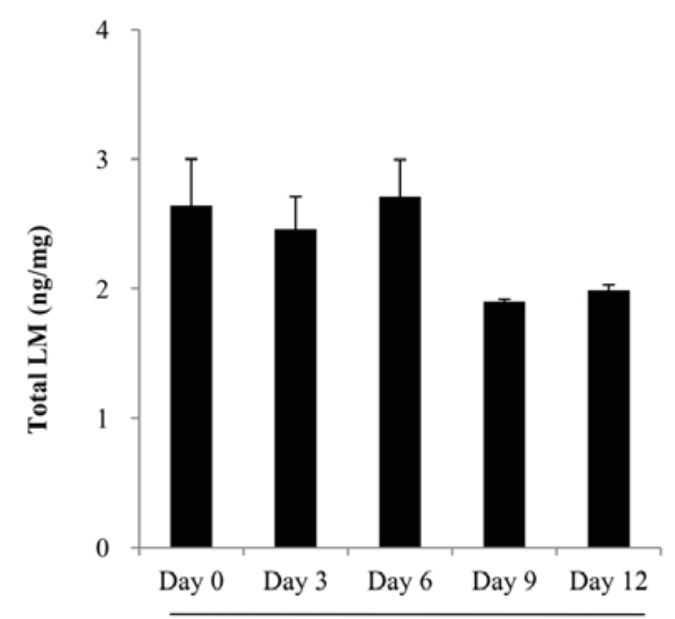

HLE B-3 BM

\section{Western blotting of 11 LM subunits in HLE B-3 BM}

B
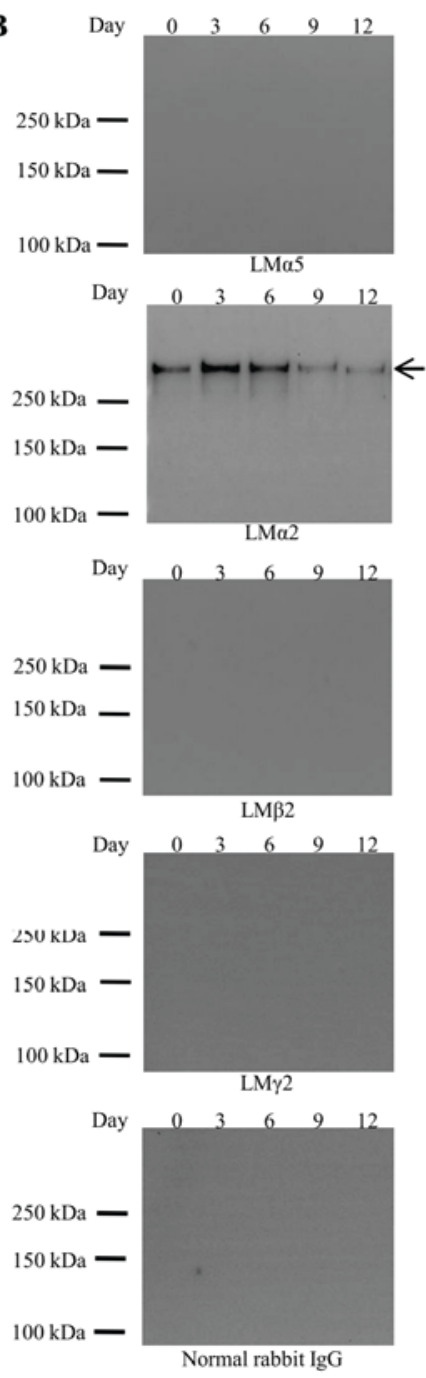

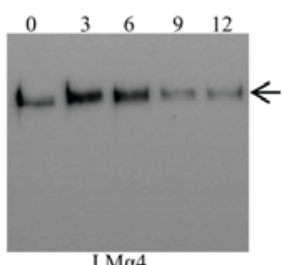

LMa4

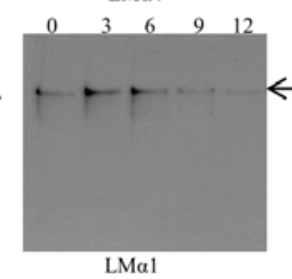

$\mathrm{LM} \alpha$
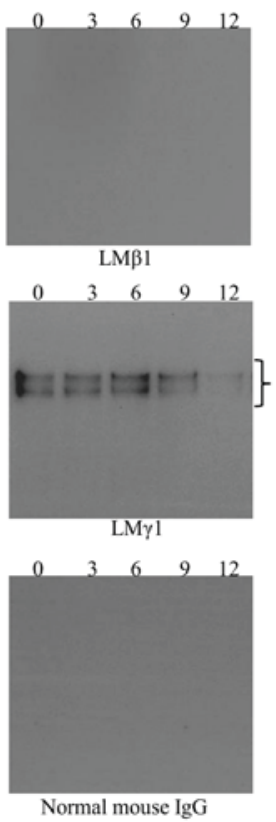

Normal mouse IgG
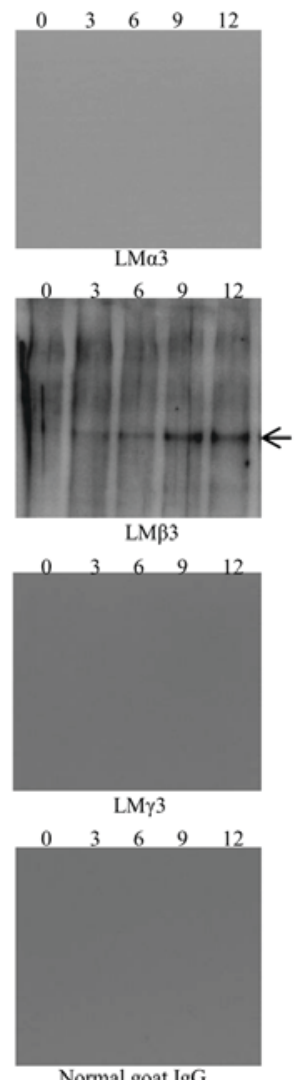

Normal goat IgG

Figure 3. LMs in HLE B-3 BMs. (A) LM levels in HLE B-3 BMs (days 0, 3, 6, 9 and 12) analyzed by LM ELISA. (B) Detection of 11 LM subunits in HLE B-3 BMs (days 0, 3, 6, 9 and 12) by western blot analysis. Arrows and braces indicate the target proteins. BM, basement membrane; LM, laminins; IgG, immunoglobulin G.

LMs in human ALCs. To investigate LM distribution in human ALCs, H\&E staining and IHC for LMs was performed. As depicted in Fig. 4A and B, LMs were primarily distributed in the ALC layer, close to the lens epithelial cells. To evaluate LM expression levels in human ALC, extracts from 2 human ALCs were examined by ELISA and western blotting with antibodies against all 11 LM subunits. In LM ELISA, total LM levels in two human ALCs (N1 and N2 samples) differed by $\sim 3$-fold (Fig. 4C), which may have resulted from the differences on age or gender. In western blotting, the N1 sample was weakly positive for $\mathrm{LM} \gamma 2$ and negative for the remaining 10 LM subunits, whereas the N2 sample was positive for LM $\alpha 4$, $\mathrm{LM} \gamma 2$ and $\mathrm{LM} \gamma 1$ subunits and negative for the remaining eight LM subunits (Fig. 4D).

\section{Discussion}

The present study provided novel information on the LM composition in HLE B-3 cells, HLE B-3 BMs and human
ALCs. The LM411 trimer existed in the HLE B-3 cell culture supernatant. Furthermore, the results of the present study clearly demonstrated that the expression pattern of LM in ALC samples was similar to the expression of BM in HLE B-3.

It has been reported that the $\mathrm{LM} \gamma 1$ subunit was present in the HLE B-3 cell lysate (29). However, whether other LM subunits exist in HLE B-3 cells remains unknown. The results of the present study demonstrated that other than the LM $\gamma 1$ subunit, nine other LM subunits, including $\mathrm{LM} \alpha 5, \mathrm{LM} \alpha 4, \mathrm{LM} \alpha 3, \mathrm{LM} \alpha 2, \mathrm{LM} \alpha 1, \mathrm{LM} \beta 3, \mathrm{LM} \beta 2, \mathrm{LM} \beta 1$ and LM $\gamma 2$ subunits, were also present in the HLE B-3 cell lysate. Compared with that in the cell lysate, the total LM level in the HLE B-3 cell culture supernatant was lower, and only 4 LM subunits, LM $\alpha 4, \mathrm{LM} \alpha 2, \mathrm{LM} \alpha 1$ and $\mathrm{LM} \gamma 1$, were detected by western blot analysis. The presence of the LM411 trimer in the HLE B-3 cell culture supernatant indicated that LM $\beta 1$ should also be expressed in the HLE B-3 cell culture supernatant. In addition, all LMs in the culture 

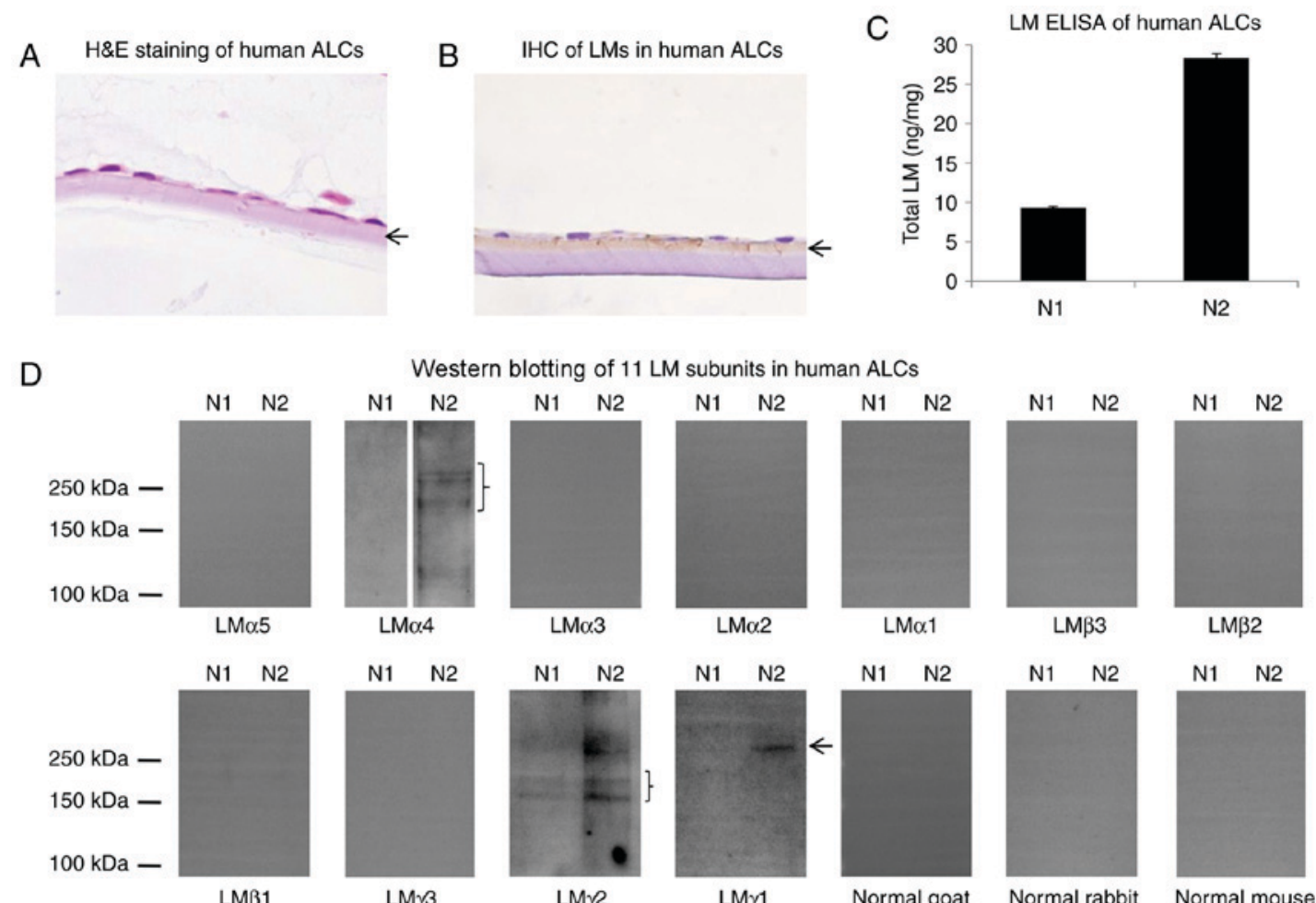

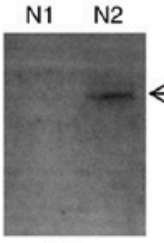

$\mathrm{LM} \gamma 1$

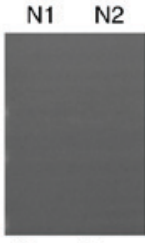

Normal goat $\lg \mathrm{G}$

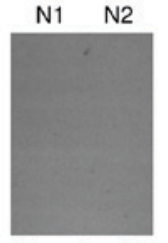

Normal rabbit $\lg \mathrm{G}$

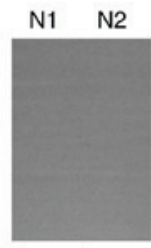

Normal mouse $\lg G$

Figure 4. LMs in human ALCs. (A) H\&E staining of human ALCs (magnification, x400). (B) IHC of LMs in human ALCs (magnification, x400 ). (C) LM ELISA in human ALCs. (D) Detection of 11 LM subunits in human ALCs by western blot analysis. N1 and N2, human ALC samples. Arrows and braces indicate the ALCs or target proteins. ALC, anterior lens capsule; H\&E, hematoxylin and eosin; IHC, immunohistochemistry; LM, laminin.

supernatant of HLE B-3 might be involved in the formation of the cell BM $(30,31)$.

In the present study, it was also demonstrated that HLE B-3 cells could synthesize BMs rich in LMs for a short period (0-6 days following 95\% confluence). HLE B-3 BMs contained $\mathrm{LM} \alpha 4, \mathrm{LM} \alpha 2, \mathrm{LM} \alpha 1, \mathrm{LM} \beta 3$ and $\mathrm{LM} \gamma 1$ subunits, which were similar to LM subunits in the HLE B-3 cell culture supernatant, except for LM $\beta 3$ and LM $\beta 1$. Considering the results of the various detection methods for the LM subunits in HLE B-3 cell lysate, culture supernatant and BMs, the LM411 trimer seems to be the major LM trimer in HLE B-3 BMs, although LM $\beta 1$ was not detected by western blot analysis.

Previous ICC studies have demonstrated that LMs are a major component of LECs and ALCs $(22,32)$, results that are consistent with the findings of the present study. Studies focusing on LM subunits in human ALCs are rare; the present study provided evidence that $3 \mathrm{LM}$ subunits (LM $\alpha 4, \mathrm{LM} \gamma 2$ and LM $\gamma 1$ ) could be detected in human ALCs by western blotting. In the present study, HLE B-3 BM was positive for $\mathrm{LM} \alpha 4, \mathrm{LM} \alpha 2, \mathrm{LM} \alpha 1, \mathrm{LM} \beta 3$ and $\mathrm{LM} \gamma 1$ subunits, which contained two LM subunits (LM $\alpha 4$ and $\mathrm{LM} \gamma 1$ ) of the three that were expressed in human ALCs. Therefore, HLE B-3 BM constructed in vitro may represent a substitute for human ALCs for future studies into LM. It is a limitation that only three human ALCs were used in the present study. Further studies using more human ALC samples for evaluation of LM expression levels are necessary to confirm the results of the present study and their similarities with the established BM model of HLE B-3.
The LM411 trimer may be the predominant LM trimer in human ALCs. It is well known that LM411 can promote cell adhesion, cell migration, angiogenesis, tumor invasion and the differentiation of stem cells (33-35). Therefore, LM411 may be involved in the progression of human LEC development, migration and transformation into lens fiber cells, and that abnormal expression of LM411 may contribute to the pathogenesis of cataracts; however, this requires further confirmation.

In previous studies, LECs were mainly used as an in vitro cell model to study the possible pathogenesis of cataracts, with the results indicating that dysfunction of LECs might serve a key role in cataract formation (8-10). To the best of our knowledge, the present study is the first to report the construction of an in vitro ALC model using human LEC cell lines. The in vitro ALC model is advantageous as it allows for the study of the contribution of BM proteins, such as LMs, on the pathogenesis of cataracts. Further studies are required to confirm the present results, validate HLE B-3 BMs as an in vitro ALC model and investigate the possible molecular pathways involving LMs in the pathogenesis of cataracts in vitro and in vivo.

In conclusion, an in vitro human ALC model rich in LMs was successfully constructed using the HLE B-3 cell line, which could be valuable for the study of the molecular biological mechanisms of cataract development and for seeking novel effective drugs for cataract treatment.

\section{Acknowledgements}

Not applicable. 


\section{Funding}

The present study was supported by grants from the Nature Science Foundation of China (no. 1470618), the Scientific Research Foundation of First Affiliated Hospital of Haerbin Medical University (no. 2017B013), the Major Program of Applied Technology Research and Development Plan of Heilongjiang Province (no. GY2016ZB0159), the Special Fund for the Doctoral Program of Higher Education (no. 20132307120035), the Natural Science Foundation of China (no. 81300728), the Natural Science Foundation of Heilongjiang Province of China (no. QC2010113) and the Postdoctoral Grant of Heilongjiang Province (no. LBH-Q12038).

\section{Availability of data and materials}

The analyzed data sets generated during the study are available from the corresponding author on reasonable request.

\section{Authors' contributions}

The paper is an original study presenting novel work that has not been published or accepted elsewhere. All authors have seen the manuscript and approved to submit to your journal. Project design: LX, TX, HT and LP; clinical samples and information collection: YY, SY and TX; project guidance: $\mathrm{QH}$ and LX; experiment and data analysis: YY, QH, JH, YH, HT, SL, WX, GH, ZH, LX and TX; manuscript revision: HT.

\section{Ethics approval and consent to participate}

All experiments were performed with the approval of the Internal Review Board of Harbin Medical University and were conducted in accordance with Declaration of Helsinki Principles. Informed consent was obtained from all patients prior to mortality or from their families following mortality for inclusion of autopsy data.

\section{Consent for publication}

Written informed consent was obtained for all patients.

\section{Competing interests}

The authors declare that they have no competing interests.

\section{References}

1. Michael R and Bron AJ: The ageing lens and cataract: A model of normal and pathological ageing. Philos Trans R Soc Lond B Biol Sci 366: 1278-1292, 2011.

2. Bourne RR, Stevens GA, White RA, Smith JL, Flaxman SR, Price H, Jonas JB, Keeffe J, Leasher J, Naidoo K, et al: Causes of vision loss worldwide, 1990-2010: A systematic analysis. Lancet Glob Health 1: e339-e349, 2013.

3. Khairallah M, Kahloun R, Bourne R, Limburg H, Flaxman SR, Jonas JB, Keeffe J, Leasher J, Naidoo K, Pesudovs K, et al: Number of people blind or visually impaired by cataract worldwide and in world regions, 1990 to 2010. Invest Ophthalmol Vis Sci 56: 6762-6279, 2015.

4. Pollreisz A and Schmidt-Erfurth U: Diabetic cataract-pathogenesis, epidemiology and treatment. J Ophthalmol 2010: 608751, 2010.

5. Roberts JE: Ultraviolet radiation as a risk factor for cataract and macular degeneration. Eye Contact Lens 37: 246-249, 2011.
6. Asbell PA, Dualan I, Mindel J, Brocks D, Ahmad M and Epstein S: Age-related cataract. Lancet 365: 599-609, 2005.

7. Rao GN, Khanna R and Payal A: The global burden of cataract. Curr Opin Ophthalmol 22: 4-9, 2011.

8. Bhat SP: The ocular lens epithelium. Biosci Rep 21: 537-563, 2001.

9. Li WC, Kuszak JR, Dunn K, Wang RR, Ma W, Wang GM, Spector A, Leib M, Cotliar AM, Weiss M, et al: Lens epithelial cell apoptosis appears to be a common cellular basis for non-congenital cataract development in humans and animals. J Cell Biol 130: 169-181, 1995.

10. Martinez $G$ and de Iongh RU: The lens epithelium in ocular health and disease. Int J Biochem Cell Biol 42: 1945-1963, 2010.

11. Schéele S, Nyström A, Durbeej M, Talts JF, Ekblom M and Ekblom P: Laminin isoforms in development and disease. J Mol Med (Berl) 85: 825-836, 2007.

12. Durbeej M: Laminins. Cell Tissue Res 339: 259-268, 2010.

13. Yurchenco PD and Patton BL: Developmental and pathogenic mechanisms of basement membrane assembly. Curr Pharm Des 15: 1277-1294, 2009.

14. Miner JH and Yurchenco PD: Laminin functions in tissue morphogenesis. Annu Res Cell Dev Biol 20: 255-284, 2004.

15. Byström B, Virtanen I, Rousselle P, Gullberg D and Pedrosa-Domellöf F: Distribution of laminins in the developing human eye. Invest Ophthalmol Vis Sci 47: 777-785, 2006.

16. Kohno T, Sorgente N, Ishibashi T, Goodnight R and Ryan SJ: Immunofluorescent studies of fibronectin and laminin in the human eye. Invest Ophthalmol Vis Sci 28: 506-514, 1987.

17. Uechi G, Sun Z, Schreiber EM, Halfter W and Balasubramani M: Proteomic view of basement membranes from human retinal blood vessels, inner limiting membranes, and lens capsules. J Proteome Res: Jul 17, 2014 (Epub ahead of print).

18. Danysh BP and Duncan MK: The lens capsule. Exp Eye Res 88: 151-164, 2009.

19. Zenker M, Tralau T, Lennert T, Pitz S, Mark K, Madlon H, Dötsch J, Reis A, Müntefering H and Neumann LM: Congenital nephrosis, mesangial sclerosis and distinct eye abnormalities with microcoria: An autosomal recessive syndrome. Am J Med Genet A 130A: 138-145, 2004.

20. Stunf S, Hvala A, Vidovič Valentinčič N, Kraut A and Hawlina M: Ultrastructure of the anterior lens capsule and epithelium in cataracts associated with uveitis. Ophthalmic Res 48: 12-21, 2012.

21. Suh J, Moncaster JA, Wang L, Hafeez I, Herz J, Tanzi RE, Goldstein LE and Guénette SY: FE65 and FE65L1 amyloid precursor protein-binding protein compound null mice display adult-onset cataract and muscle weakness. FASEB J 29: 2628-2639, 2015.

22. Joo CK, Lee EH, Kim JC, Kim YH, Lee JH, Kim JT, Chung KH and Kim J: Degeneration and transdifferentiation of human lens epithelial cells in nuclear and anterior polar cataracts. J Cataract Refract Surg 25: 652-658, 1999.

23. de Iongh RU, Wederell E, Lovicu FJ and McAvoy JW: Transforming growth factor-beta-induced epithelial-mesenchymal transition in the lens: A model for cataract formation. Cells Tissues Organs 179: 43-55, 2005.

24. Arita T, Murata Y, Lin LR, Tsuji T and Reddy VN: Synthesis of lens capsule in long-term culture of human lens epithelial cells. Invest Ophthalmol Vis Sci 34: 355-362, 1993.

25. Hirako Y, Yonemoto Y, Yamauchi T, Nishizawa Y, Kawamoto Y and Owaribe $\mathrm{K}$ : Isolation of a hemidesmosome-rich fraction from a human squamous cell carcinoma cell line. Exp Cell Res 324: 172-182, 2014.

26. Li X, Qian H, Sogame R, Hirako Y, Tsuruta D, Ishii N, Koga H, Tsuchisaka A, Jin Z, Tsubota K, et al: Integrin $\beta 4$ is a major target antigen in pure ocular mucous membrane pemphigoid. Eur J Dermatol 26: 247-253, 2016.

27. Andjelic S, Drašlar K, Hvala A and Hawlina M: Anterior lens epithelium in cataract patients with retinitis pigmentosa-scanning and transmission electron microscopy study. Acta Ophthalmol 95: e212-e220, 2017.

28. Li X, Qian H, Takizawa M, Koga H, Tsuchisaka A, Ishii N, Hayakawa T, Ohara K, Sitaru C,Zillikens D, et al: N-linked glycosylation on laminin $\gamma 1$ influences recognition of anti-laminin $\gamma 1$ pemphigoid autoantibodies. J Dermatol Sci 77: 125-129, 2015.

29. Patrick B, Li J, Jeyabal PV, Reddy PM, Yang Y, Sharma R, Sinha M, Luxon B, Zimniak P, Awasthi S, et al: Depletion of 4-hydroxynonenal in hGSTA4-transfected HLE B-3 cells results in profound changes in gene expression. Biochem Biophys Res Commun 334: 425-432, 2005. 
30. Sigle RO, Gil SG, Bhattacharya M, Ryan MC, Yang TM, Brown TA, Boutaud A, Miyashita Y, Olerud J and Carter WG: Globular domains $4 / 5$ of the laminin alpha3 chain mediate deposition of precursor laminin 5. J Cell Sci 117: 4481-4494, 2004.

31. Frank DE and Carter WG: Laminin 5 deposition regulates keratinocyte polarization and persistent migration. J Cell Sci 117: 1351-1363, 2004.

32. Zhang XH, Sun HM and Yuan JQ: Extracellular matrix production of lens epithelial cells. J Cataract Refract Surg 27: 1303-1309, 2001.

33. Gonzalez AM, Gonzales M, Herron GS, Nagavarapu U, Hopkinson SB, Tsuruta D and Jones JC: Complex interactions between the laminin alpha 4 subunit and integrins regulate endothelial cell behavior in vitro and angiogenesis in vivo. Proc Natl Acad Sci USA 99: 16075-16080, 2002.
34. Patarroyo M, Tryggvason $\mathrm{K}$ and Virtanen I: Laminin isoforms in tumor invasion, angiogenesis and metastasis. Semin Cancer Biol 12: 197-207, 2002.

35. Qu H, Liu X, Ni Y, Jiang Y, Feng X, Xiao J, Guo Y, Kong D, Li A, $\mathrm{Li}$ X, et al: Laminin 411 acts as a potent inducer of umbilical cord mesenchymal stem cell differentiation into insulin-producing cells. J Transl Med 12: 135,2014.

cc) (1) (9) This work is licensed under a Creative Commons Attribution-NonCommercial-NoDerivatives 4.0 International (CC BY-NC-ND 4.0) License. 